\title{
SEMIOTICALly ARTICULATED MUlTIMEDIA CODING MODELS
}

\author{
Ruben Gonzalez \\ School of Information Technology, Griffith University, \\ PMB 50, Gold Coast Mail Centre, QLD, 4217 \\ Email: R.Gonzalez@gu.edu.au
}

\begin{abstract}
Compression has been the sole objective of traditional audio and visual data coding schemes. More recently the requirements for effective information management have focused attention on the question of what mechanisms are required to support content based retrieval. This has been largely approached as a separate problem by either using index files containing meta-data or attempting to exploit whatever information is readily available in existing compressed data streams. This paper will review existing support for information management in current coding models, and contrast this with the requirements for effective retrieval. Additionally, a class of semiotically based coding models will be presented which are equally suited for achieving both high compression and effective information management.
\end{abstract}

\section{INTRODUCTION}

With the advent of multimedia systems it suddenly became possible to store, manipulate and experience large amounts of both audio and visual information on a single platform. This was brought about through two main developments. On one hand computers became capable of rendering high quality audio and video in real-time while simultaneously compression techniques had matured to the point where storage and bandwidth limitations were unimportant. The large availability of compressed audio-visual data as however has created its own set of information management problems [1].

Unlike text, with audio-visual data it is not possible to find and retrieve a document based on a full-text search of its content. A problem exists when trying to locate audio or visual data in a large data set and one can only remember some of its characteristics such as the tune of a musical composition but not its name or an image when one can only describe it abstractly. Content based retrieval techniques attempt to overcome this problem and provide support for advanced multimedia information management.

The traditional approach is to provide additional information about the content (metadata) in the form of manual annotations. This annotation information is stored separately from the coded data stream in an index. As a separate entity, some agent is required to associate or bind the annotations to the coded data before the information management system can intelligently interact with the data (figure 1). Efforts in generating the annotation data automatically have been based on either constrained recognition or statistical attributes of the data. Both processes typically require full decoding of the compressed data before they can be applied.

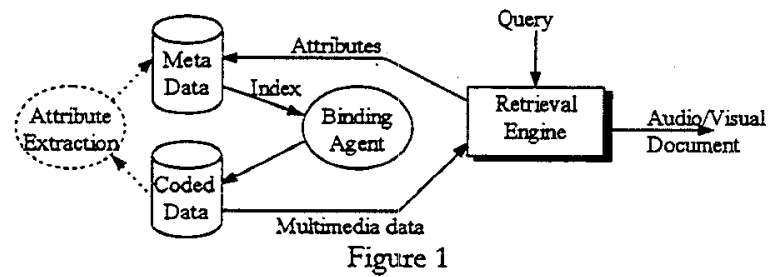

An altemative approach presented in this paper is to build support for information management into the compressed data by using articulated representations. All advanced animal communication is articulated and a distinguishing feature of human communication is the principle of double articulation that specifies a two level communication structure [2]. Exploiting this articulation becomes pivotal for effective information management.

Semiotics is the study of articulation in communication and is concerned with the relationships of meaning of between signs. Apart from the classical verbal and lexical communication processes to which it is applied, semiotics is pertinent to a wider range of interactive information processing. Morris [3] describes semiotics in the context of three basic phases of interaction. The first stage is the perceptual stage which is based on seeking signs or objects. Then, the manipulatory stage is gaining control of the signs followed by the consumatory stage which lets the signs perform their function. In the perceptual stage the signs are primarily designative in that they signify what to expect from them. In the manipulatory stage, signs are prescriptive because they signify appropriate courses of action. In the consumatory stage, signs are appraisive since they reveal how well they respond to the desired manipulation.

\section{EXISTING WORK}

The existing approach to managing coding multimedia data is based on either recognition or statistical similarity measures. In either case the management revolves around the index of captions, labels composed of statistical attributes or other such meta-data generated separately from the data itself. This approach constrains the information management because it treats the coded data 
as simply "renditions" of some external entity in the sense of the classic E-R model. Support for information management is totally external to the data itself and based on the antecedently generated indices.

Since the indices and data are normally separate, support for information management is not intrinsic to the data but instead specific to some application. This creates a problem with portability and reusability of the data requiring the generation of new indices whenever the data is reused in a new environment or system. In this context, this limitation can only be overcome by appending or interleaving the index into the data stream as well as embedding the software query engine [4]. In any case the actual multimedia data representation remains unchanged, that is unstructured. Additionally, the interleaved meta-data is specific to the application context, and potentially of limited use in new contexts.

The statistically based retrieval methods preclude semantic retrieval since they can only generate low level, analytic labels. These are unstructured and can not convey any meaning about the data. Altematively, the semantic methods require a constant need for knowledge about the definition of new objects and their properties to contend with unfamiliar environments. Being equivocal and computationally intensive, these work successfully only under highly constrained conditions. These current methods permit little or no exploitation of the natural articulation in the data itself. Excepting in highly constrained environments (e.g. [5]), syntactic or structural methods have been largely overlooked as the basis of information management. The use of suitably abstracted structural over statistical information is cognitively more appropriate for similarity matching [6] and it is less constrained than semantic methods.

The current deficiencies in multimedia information systems are due to the underlying coding models not being able to directly support information management. These models generally have only a single level of articulation and are only mono-symbolic. This precludes the models from being able to convey any significant information. While some of these symbols (or units) support limited parametisation their uniformity makes it impossible to extract meaningful information from the models. The articulatory units in coding schemes which are shown in tables 1,2 and 3 respectively.

\begin{tabular}{|c|l|}
\hline Articulatory Units & \multicolumn{1}{c|}{ Intraframe Coding Schemes } \\
\hline Pixels & PCM / Statistical / Predictive \\
\hline 1D Vectors & Run length Encoding, WBS, etc \\
\hline Flat Polygons & 2D RLE / Quadtrees / etc \\
\hline Smooth Polyongs & VQ / Transform / Contour Texture \\
\hline 3D Objects & Model Based Coding \\
\hline
\end{tabular}

Table I. Image Coding Schemes by Articulation
Articulatory Units Interframe Coding Schemes

\begin{tabular}{|c|l|}
\hline Still Images & None \\
\hline Stationary Change & Conditional Replenishment \\
\hline Planar Motion & Motion Compensation \\
\hline Layered Motion & Object-Background Schemes \\
\hline Full 3D Motion & 3D Model Based Coding \\
\hline
\end{tabular}

Table 2. Video Coding Schemes by Articulation

Articulatory Unit:

Coding Schemes

Amplitude Variations PCM, Temporal Predictive

Frequency Bands/Bins $\quad$ ATC, subband, spectral VQ

\begin{tabular}{l|l}
\hline Frequency tracks & Simusoidal Transform
\end{tabular}

\begin{tabular}{l|l} 
Harmonic groups & Direct Harmonic Coding
\end{tabular}

Table 3. Audio Coding Schemes by Articulation

For example, quadtrees are mono-symbolic, n-level articulations, but the elements are all regular squares of fixed sizes occurring in restricted configurations. Virtually in all cases, both the characteristics of the model elements and the possible relationships between the elements are heavily constrained. This is due to the heavy regularisation of the data performed by compression algorithms to enhance codability. The granularity of the elements is also often too small for them to be of any significance. In addition to model based limitations the complexity of the representations derived from these models tend to obfuscate any salient properties of the encoded data.

The support for structure based coding in the upcoming MPEG 4 standard is based on arbitrary shape based coding of semantic objects. This however requires a separate alpha channel transparency mask to define the outline of each object. Apart from typically requiring manual generation of the mask itself which is a semantic process, the mask storage incurs additional overheads.

Researchers in semiotics have proposed a number of articulations for multimedia data [2] as the basis for semiosis, composed of sets of signs and subsigns. While the signs are semantic objects the subsigns are merely structural elements, from which the signs are composed.

\section{NEW CODING MODELS}

The identifying feature of any compression system is its coding model. This determines the capabilities of the system by defining the nature of its elemental components and defining or delimiting the relationships and interactions both between and with these components. For any given coding model various distinct representations may be feasible. The representation scheme determines the accessibility to the elemental components, compaction, interactive manipulability and the decoding complexity of the compressed data.

The difference between a coding model and its representation is that the model specifies $w h a t$ the elements are in terms of which the data is to be encoded and its organisation. The representation determines bow these elements are encoded. For example, given a coding model 
consisting of a collection of smooth curves, each curve may be represented as either a list of polynomial coefficients, a chain code, or a string of coordinates. Alternatively, in the specific case of the coefficients, these may be stored as either scaled integers, normalised rational numbers, or even written out textually.

This work attempts to apply the existing efforts in semiotics to develop new articulated coding models for multimedia data. For effective information management the articulatory units in the final representation must be also be directly and randomly accessible, individually decodable and uniquely identifiable. Few existing coding schemes support randomly accessible or individually decodable elements. The coding models should also exhibit some form of data abstraction.

From cognitive psychology we know that cognitive objects are mentally described in terms of their defining and characteristic attributes separately [7]. Each articulatory unit in the coding model must therefore be composed at the very least of a defining and possibly multiple characteristic attributes. In this case the defining attribute is the type of the element. The characteristic attributes are additional properties used to parametise the generic element types. In addition, relationships among stimuli are often more important than the nature of the stimuli themselves. Hence, each element should also be described by its relational attributes, where the defining, characteristic and relational attributes are orthogonal to each other. These elements are sub-semantic entities which must be grouped to form meaningful objects.

The proposed articulatory elements are congruent with the nature of the analysis performed by precognitive psychophysical processes. For each media type the articulation consists of three different types of units as shown in Figure 1, the formal definition of these models may be found in [8].
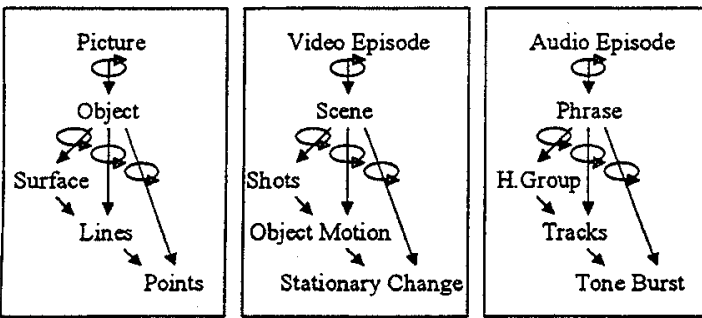

Figure 1. Semiotic Articulations

Information management is directly supported in coded data in the form of generalised scene descriptions which emerge from considering only the information encapsulated by the articulation, expressed in terms of the defining and relational attributes. Altematively, conventional statistical similarity based retrieval may be supported if the characteristic attributes only are considered, since each element readily conveys statistical information about its characteristics. Table 4 shows the characteristic attributes associated with each articulatory unit. Recognition of select groupings having specific semantic interpretations may be performed.

\begin{tabular}{|c|c|c|c|}
\hline Definings & \multicolumn{3}{|c|}{ Characteristic Attributes } \\
\hline plyel: & Colour & Location & - \\
\hline Wine & $\begin{array}{l}\text { Path (length, } \\
\text { orientation) }\end{array}$ & $\begin{array}{l}\text { Intensity } \\
\text { profile }\end{array}$ & - \\
\hline Surface & $\begin{array}{l}\text { Shape (area, } \\
\text { orientation) }\end{array}$ & $\begin{array}{l}\text { Intensity } \\
\text { profile }\end{array}$ & Texture \\
\hline $\begin{array}{l}\text { Stationary } \\
\text { change }\end{array}$ & $\begin{array}{c}\text { Region Shape } \\
\text { Area }\end{array}$ & $\begin{array}{l}\text { Region } \\
\text { Content }\end{array}$ & $\begin{array}{c}\text { Time / } \\
\text { Duration }\end{array}$ \\
\hline $\begin{array}{l}\text { Object: } \\
\text { Motion }\end{array}$ & $\begin{array}{c}\text { Affine } \\
\text { Transformation }\end{array}$ & Depth Order & $\begin{array}{l}\text { Time / } \\
\text { Duration }\end{array}$ \\
\hline Shot & Camera Motion & $\begin{array}{l}\text { Lighting } \\
\text { Changes }\end{array}$ & $\begin{array}{l}\text { Time / } \\
\text { Duration }\end{array}$ \\
\hline Pure Tone & Frequency & Duration & Intensity \\
\hline $\begin{array}{l}\text { Frequency } \\
\text { Track }\end{array}$ & $\begin{array}{l}\text { Pitch Contour } \\
\text { (melody) }\end{array}$ & Modulation & $\begin{array}{l}\text { Intensity } \\
\text { Contour }\end{array}$ \\
\hline Tharmonic & $\begin{array}{l}\text { Frequency } \\
\text { spacing }\end{array}$ & $\begin{array}{l}\text { Energy } \\
\text { distribution }\end{array}$ & $\begin{array}{l}\text { Chord } \\
\text { Contour }\end{array}$ \\
\hline
\end{tabular}

\section{ONGOING WORK}

The ongoing work is that of developing fully interactively manipulable, compressed representations for audio and video data based on these semiotically articulated coding models. Part of this work entails the development of robust grouping algorithms to merge the lower articulatory sub-units into more meaningful semiotic signs. In the case of images this aspect of the work is reported in the paper by Wardhani and Gonzalez [9]. Our structured audio work is reported in a separate paper by Melih, Gonzalez and Ogunbona [10].

Our compact representation for structured video extracts the intra-frame primitive elements from each level in succeeding stages. The encoder initially attempts to approximately fit the surfaces which are the largest primitives, to the underlying; frame data. The encoding process then proceeds by attempting to fit the next lower level, smaller primitives to the residual data. The adequacy of the fit is based on estimating the cost of encoding the same data using a coplanar arrangement of lower order primitives alone versus a layered representation. If no coding gain is achieved through encoding the region as the high order primitive plus the required lower order primitives then it is discarded and the region encoded with the lower order primitives alone. This provides a multiresolution representation since each layer consists of primitives of different spatial/temporal/spectral resolution.

The representation for a layer becomes a layer type specifier followed by the number of different colour groups, and each colour group. Each colour group is defined as the colour for the group, the number of elements in the group and the array of elements. Each 
element is defined by its spatial relationship with its preceding element and any additional shape information. The resulting data is then variable length coded. In the interframe coding only stationary changes are modeled.

While maintaining relatively high image quality this scheme can achieve compressed ratios on par with conventional schemes. Depending on the amount of motion in the video the ratios between $20: 1$ to $80: 1$ compression are achived. The data rates for QCIF (176) 144 pixels) teleconferencing type colour video are about $2 \mathrm{k}$ bytes per frame. The subjective quality is on par or better than most existing software decodeable video coding schemes. Due to the nature of the compressed representation faster then realtime decoding is possible on even low end PCs. Figure 2 shows the progressive update of both a full image and a conditional replenishment image.

The versatility of the representation for content based retrieval is demonstrated by the fact that scene changes can be detected simply by a combination of thresholding the number of primitive elements per frame and the change in colour composition given by the colour fields. At the most, this only involves integer addition of a few of the data fields explicitly given in the representation. Common statistical attributes such as the average image colour can simply be obtained from the number of elements in each colour group. The texture is given by the type of the elements and their colour contrast in any given region. Additionally, distinctly coloured objects can be directly isolated and manipulated by simply identifying the colour group(s) to which they belong.

\section{CONCLUSIONS}

This paper has discussed the role of articulated coding models for generating compressed, structured audio and video data representations that provide intrinsic support for information management \& interactive manipulation. Due to its role in cognition, semiotic articulation is the natural basis for structured data representations. The existing coding schemes which produce unstructured representations are unable to readily support the information management, instead obstructing the management of the data. The current separate index based solutions suffer from lack of portability, introducing significant storage overheads, limited flexibilty, and the indexed data is still not interactvely manipulable.

In addition to proposing semiotically articulated coding models and the required characteristics of the resultant data representations, this paper has described a structured video compression scheme which directly supports information management. Future work includes developing a representation which fully implements the model, as well as developing automatic methods for structural analysis and grouping of the articulatory units to support higher level queries.

\section{REFERENCES}

[1] M.H.O'Docherty, C.N.Daskalakis, "Muitimedia Information Systems - The Management and Semantic Retrieval of all Electronic Data Types.", The Computer Joumal, Vol.34, No.3, 1991, pp.225-238.

[2] W. Nöth, Handbook of Semiotics, Indiana Univ. Press 1990

[3] C.Morris, Signification and Significance, MIT Press, 1964.

[4] P.Senthil Kumar, G.Phanendra Babu, "Intelligent Multimedia Data: Data + Indices + Inference", Multimedia Systems Jouraal

[5] J.KWu, Y.H.Ang, P.C.Lam, S.KMoorthy, A.D.Narasimbalu, "Facial Image Retrieval, Identification, and Inference System.", Proceedings of ACM Multimedia 93, August 1993, Pp.47-53.

[6] A.Tversky, "Features of Similarity", Psychological Rerriew, Vol.84, No.4, July 1977, pp.327-352.

[7] M.W.Eysenck, M.T.Keane, Cognitive Psychology - A Students Handbook, Lawrence Erlbaum Associates, 1990

[8] R.Gonzalez, "HyperMedia Data Models, Coding and Semiotics," PIEEE, July 1997, Vol.85, No.7, pp.1111-1140.

[9] A.V.Warhani and R.Gonzalez, "Using High Level Information for Region Grouping.". Proceedings of TENCON'97, Brisbane, December 1997.

[10] KMelih, R.Gonzalez and P.Ogunbona, "An Audio Representation for Content Based Retrieval" Proceedings of TENCON'97, Brisbane, December 1997.

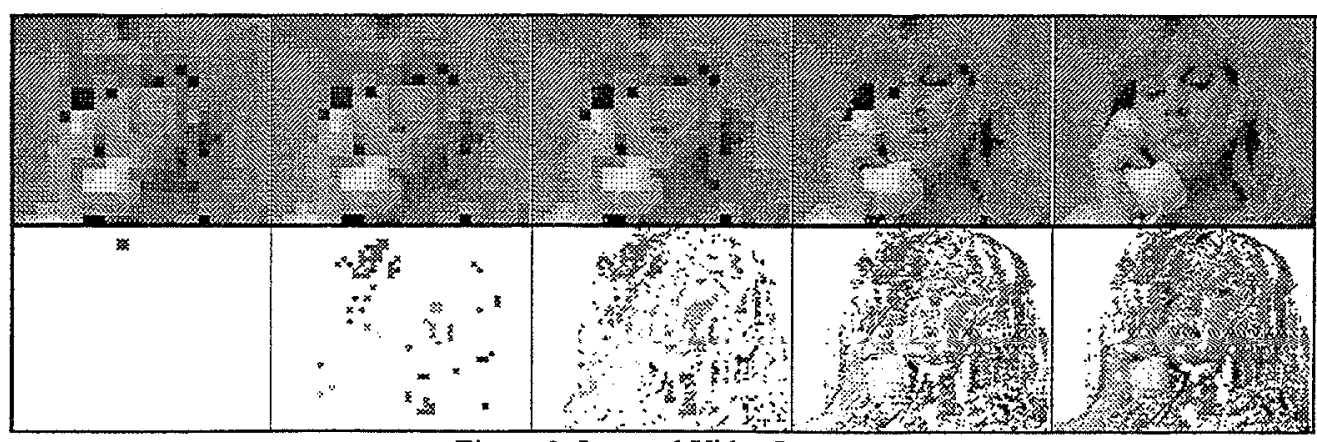

Figure 2. Layered Video Images 